\title{
Motivación de logro, indicadores de competitividad y rendimiento en un equipo de jugadores de fútbol de competición varones entre 14 y 24 años*
}

Achievement motivation, competitiveness and sports performance in a team of sportsmen soccer players between 14 and 24 years old

Recibido: abril 17 de 2010 | Revisado: mayo 14 de 2010 | Aceptado: mayo 20 de 2010

\author{
Alejo García-NaveirA ** \\ Centro de Estudios e Investigación, Club Atlético \\ de Madrid, España \\ EDUARDO REMOR ${ }^{* * * *}$ \\ Universidad Autónoma de Madrid, España
}

SICI: 1657-9267(201108)10:2<477:MLICYR>2.0.TX;2-Y

Para citar este artículo. García-Naveira, A. \& Remor, E. (2011). Motivación de logro, indicadores de competitividad y rendimiento en un equipo de jugadores de fútbol de competición varones entre 14 y 24 años. Universitas Psychologica, 10 (2), 477-487.

* Artículo de investigación.

** C/ Jordán, 15. Puerta derecha. San Lorenzo de El Escorial, 28200 Madrid, España. E-mail: alejopsico@europe.com

*** C/ Ivan Pavlov, 6, Universidad Autónoma de Madrid, 28049 Madrid, España. E-mail: eduardo.remor@uam.es

Research-ID: Remor, E., B-7133-2011.

\section{RES U MEN}

Para identificar hasta qué punto el rasgo de competitividad está relacionado con el rendimiento deportivo en el fútbol, y en qué medida la edad y la categoría deportiva pueden influir sobre estas variables, se ha diseñado un estudio descriptivo-correlacional. Las variables edad, categoría deportiva, rendimiento deportivo, motivación de éxito $(\mathrm{Me})$, motivación para evitar el fracaso (Mef) y competitividad rasgo han sido recogidas en 151 futbolistas varones (entre 14 y 24 años) de un club deportivo madrileño (España). Los resultados indicaron que el rendimiento deportivo asciende de acuerdo con la edad. Consecuentemente, se ha observado una relación directa entre la categoría deportiva y el rendimiento. La Me, Mef y competitividad rasgo se han asociado con el rendimiento y han variado en función de la categoría deportiva. No se ha observado una relación entre la Me, Mef y la competitividad rasgo con la edad de los deportistas.

Palabras clave autores

Competitividad, motivación, alto rendimiento, deporte, fútbol.

Palabras clave descriptores

Psicometría, test psicológico, grupo de edad, comportamiento de grupo, actividad sensomotriz, voluntad de realización.

\section{A B S T R A C T}

In order to identify to what extent competitiveness trait is related to sport performance in soccer, and to what extent the age and sport category can influence these variables, a descriptive cross-sectional study has been developed. The variables age, sport category, sport performance, achievement motivation (Me), motivation to avoid the failure (Mef) and competitiveness trait have been assessed in 151 men soccer players (between 14 and 24 y.o.) of a Spanish sport club. The results indicated that the sport performance ascends with age. Consequently, a direct relationship between the sport category and the performance has been observed. Me, Mef and competitiveness trait have been associated with the performance and has varied based on the sport category. No correlation between Me, Mef, competitiveness and age of the sportsmen has been found.

Key words authors

Competitiveness, Motivation, High Performance, Sports, Soccer

Key words plus

Psychometrics, Psychological Tests, Group Behaviour, Sensorimotor Activities, Achievement Motivation. 


\section{Introducción}

De acuerdo con Carlstedt (2004), Jarvis (2005) y Moran (2004) en el deporte, en general, los componentes psicológicos y emocionales trascienden los aspectos puramente físicos, técnicos y tácticos de la ejecución deportiva. Dicho de otra forma, tanto el éxito como el fracaso de un deportista proceden de una combinación de capacidades condicionales físicas (p. ej. fuerza, velocidad y resistencia), técnicas (p. ej. golpeo y control del balón), tácticas (p. ej. realizar un repliegue y jugada a balón parado) y psicológicas (p. ej. personalidad y motivación). Partiendo de esta reflexión, diferentes autores han investigado cuáles variables psicológicas intervienen en el rendimiento y éxito deportivo.

Por ejemplo, entre los temas más investigados en Psicología del Rendimiento Deportivo está el estudio de la personalidad de los deportistas (Dosil, 2003; Singer, Hausemblas \& Jannelle, 2001; Vealey, 1992, 1994; Viadé, 2003). Entender, explicar y predecir la conducta deportiva a través de la personalidad del deportista ha sido el punto de partida de muchas investigaciones (p. ej.: Núñez, 1998; Ruiz, 2006). Dentro de un abanico de posibilidades de estudio, la medición del rasgo de competitividad es una variable importante para comprender y predecir la conducta deportiva (Houston, Harris, Moore, Brummett \& Kmetani, 2005; Remor, 2007). En este sentido, Weinberg y Gould (1996) indican que la competitividad es considerada, por algunos entrenadores, como uno de los rasgos más deseables en los deportistas. Los individuos que son descritos como "muy motivados" o "competitivos" por sus entrenadores, tienden a presentar un alto nivel de esfuerzo, son perseverantes en conseguir la victoria, juegan duro y rara vez se dan por vencidos. Este tipo de deportistas suelen tener un mejor rendimiento que sus iguales, en parte, a causa de su actitud competitiva.

\section{Definición de Competitividad}

Martens (1975) definió la competitividad como una disposición para esforzarse en satisfacer un estándar de excelencia, cuando se hacen compa- raciones en presencia de evaluadores externos. Houston, Harris, McIntire y Francis (2002) definieron la competitividad desde una perspectiva multidimensional, que hace referencia, por una parte, a la idea de que la competitividad sirve para "validar la superioridad de uno sobre la inferioridad de otros" en el deporte y, por otra, para reflejar el éxito personal y la satisfacción con este éxito, dando énfasis a los beneficios de la competitividad (por ejemplo, sentirse competente en la actividad). En definitiva, la competitividad se constituye como una conducta de logro en un contexto competitivo, donde la evaluación social es un componente clave (Remor, 2007).

A partir de la experiencia, el individuo aprende cuáles actitudes y conductas son más apropiadas (o reforzadas) dentro de su entorno, y qué resultados o consecuencias obtiene. Por lo tanto, cabe esperar diferencias en el rasgo competitividad en función del nivel competitivo del entorno y la edad del deportista.

Los deportistas de alto nivel se han ido desarrollando en un medio selectivo como la competición, donde los deportistas más competentes suelen ir consiguiendo metas deportivas (éxitos). En este sentido, la conducta de logro necesaria para superar a los rivales y conseguir el éxito en presencia de una alta evaluación social (jueces, público, familiares, cuerpo técnico y demás jugadores) se traduce en el rasgo de competitividad.

\section{Competitividad y rendimiento deportivo}

Si consideramos las propuestas de Franken, Hill y Kierstead (1994) y Weinberg y Gould (1996), las razones que llevan a un individuo a persistir en la especialización y profesionalización deportiva podría estar motivada por su rasgo de competitividad, pues cabe esperar que las personas altamente competitivas tiendan a buscar situaciones de competición y estén motivadas para obtener éxito en ellas, en comparación con personas con bajo nivel de competitividad. Esta hipótesis se ve contrastada en una serie de estudios que se presentan a continuación. 
El estudio de Gill (1986) observó, en una muestra de 237 estudiantes que participaban en clases de educación física con actividades competitivas y no competitivas, que aquellos que participaban en actividades de competición eran más competitivos que los deportistas que no participaban en dichas actividades de acuerdo con las puntuaciones en la dimensión de competitividad del Work and Family Orientation Questionnaire.

Martin y Ecklund (1994) aplicaron el Sport Orientation Questionnaire (SOQ) a una muestra de 80 corredores de fondo ( 58 hombres y 22 mujeres), con edades entre 10 y 61 años. Los corredores fueron repartidos en dos grupos en función de los tiempos personales obtenidos en las competiciones. Los deportistas del grupo rápidos (con mejores marcas deportivas) eran más competitivos que los deportistas del grupo lentos (con peores marcas deportivas).

Gill, Williams, Dowd, Beaudoin y Martin (1996), identificaron que los deportistas que participaron en eventos deportivos de competición presentaban mayores puntuaciones en competitividad (SOQ) que aquellos que no habían participado en ellos.

La investigación longitudinal de Hellandsig (1998), en diferentes deportes de equipo, aportó evidencias de que el alto rendimiento (high performance) se ha podido predecir a través de las altas puntuaciones en competitividad y competencia física autoinformada, partiendo de la edad de 15 16 años hasta la edad de 17-18 años.

Gould, Dieffenbach y Moffett (2002) entrevistaron a 10 campeones olímpicos, evaluando sus atribuciones respecto al éxito deportivo y concluyeron que la competitividad es una variable que caracteriza a los deportistas de élite.

Gee, Dougan, Marshall y Dunn (2007) han realizado un estudio longitudinal, durante 15 años, sobre personalidad y rendimiento deportivo. Para ello, evaluaron la personalidad de 124 jugadores adultos de jóckey sobre hielo de la Nacional Hockey League, durante la temporada 1991-1992, llevando a cabo un seguimiento sobre su evolución deportiva hasta finales de la temporada 2005-2006.
Varios de los rasgos dominantes de la personalidad (por ejemplo, competitividad, orientación del equipo, confianza en sí mismo, disposición analítica) fueron encontrados como indicadores significativos del rendimiento deportivo total de los jugadores.

Sáenz-López, Jiménez, Jiménez e Ibáñez (2007) entrevistaron a 14 jugadoras de la Selección Nacional de Baloncesto. En dicho estudio, la disposición para competir y el deseo de ganar (motivación de logro) fueron identificados como aspectos psicológicos indispensables según el autoinforme de las jugadoras.

Remor (2007), analizó la relación entre competitividad rasgo y éxito en la competición, mediante un experimento. El rasgo de competitividad se evaluó con el cuestionario Competitividad-10, en una muestra de 153 deportistas universitarios españoles, de ambos sexos, con una edad comprendida entre los 19 y 32 años. Los resultados indicaron que los participantes con éxito en la competición simulada en el experimento, correspondían a aquellos con mayor puntuación obtenida en el cuestionario de competitividad rasgo. Así mismo, las puntuaciones en el cuestionario se relacionaban con la autopercepción subjetiva de los participantes sobre su nivel de competitividad.

Posteriormente, García-Naveira y Remor (2008) evaluaron el rasgo de competitividad a una muestra de 61 futbolistas ( 26 de competición y 35 amateurs), todos varones, con edades entre los 20 y 24 años. Los resultados indicaron diferencias significativas en la competitividad en función del nivel de profesionalización de los deportistas, es decir, los futbolistas de competición han obtenido mayores puntuaciones en la dimensión motivación de éxito y rasgo de competitividad, y menores puntuaciones en la dimensión motivación para evitar el fracaso que los deportistas amateurs.

En general, los trabajos revisados señalan que hay una relación entre el nivel de competitividad, o las características personales asociadas a la competitividad, y el rendimiento deportivo, en términos de participación en actividades competitivas o relacionadas con el alto rendimiento deportivo. 


\section{Competitividad y edad}

Aunque fuera de la investigación, en el ámbito deportivo, hay algunas evidencias de que hay una relación entre competitividad y edad (Cashdan, 1998), son pocos los estudios que analizan la relación entre competitividad y edad en deportistas de competición.

Según Martin y Ecklund (1994), es posible que el rasgo competitividad varíe de forma curvilínea en función de la edad de los deportistas, con un aumento a lo largo de la niñez, juventud e inicio en la vida adulta, y un descenso en el final de la vida adulta y vejez. No obstante, los estudios empíricos revisados no apuntan una relación significativa entre nivel de competitividad y edad de los deportistas.

Por ejemplo, el estudio de Beaudoin (2006) con mujeres futbolistas profesionales, entre 18 y 45 años, no observó una relación significativa entre competitividad (medida por el SOQ) y edad de las deportistas. Por otra parte, el estudio de Remor (2007) con deportistas universitarios jóvenes no profesionales, no identificó una asociación significativa entre edad y la competitividad rasgo (medida por el Competitividad-10).

Teniendo en cuenta que diferentes trabajos presentan la motivación de logro y el rasgo de competitividad como una variable importante en la comprensión y predicción del rendimiento deportivo, la presente investigación se propone a: 1) analizar la posible relación entre el rasgo competitividad (y sus dimensiones) y el rendimiento deportivo y, 2) estudiar las posibles diferencias en el rasgo competitividad en función del nivel de profesionalización (categoría deportiva) y la edad de los deportistas.

\section{Método}

\section{Participantes}

La muestra está constituida por 151 futbolistas varones, integrados en un total de 7 equipos de competición (entrenan 5 días a la semana en sesiones de 2 horas) de un club deportivo de la comunidad de Madrid, con edades entre 14 y 24 años $(M=$ $17, D T=2,3)$. Los jugadores están repartidos en 2 equipos cadetes (categoría autonómica), 3 equipos juveniles (categorías Liga Nacional y División de Honor) y 2 equipos de categoría absoluta (Tercera y Segunda División "B"). En la Tabla 1, se describen las características de los jugadores en función de la categoría, equipo y edad.

TABLA 1

Distribución de categorías de equipo y edades correspondientes

\begin{tabular}{|c|c|c|c|c|c|}
\hline $\begin{array}{l}\text { Categorías } \\
\text { de equipo }\end{array}$ & Equipo & $N$ & $\%$ & $\begin{array}{l}\text { Edad } \\
\text { Media } \\
(D T)\end{array}$ & $\begin{array}{c}\text { Edad } \\
\text { Media } \\
(D T)\end{array}$ \\
\hline \multirow{2}{*}{$\begin{array}{l}\text { Cadete } \\
(n=43)\end{array}$} & $\begin{array}{l}\text { Cadete } 1 \\
\text { Autonómica }\end{array}$ & 22 & 14,6 & $\begin{array}{l}14,4 \\
(0,5)\end{array}$ & $\begin{array}{l}14,9 \\
(0,2)\end{array}$ \\
\hline & $\begin{array}{l}\text { Cadete } 2 \\
\text { Autonómica }\end{array}$ & 21 & 13,9 & & $14(0)$ \\
\hline \multirow{3}{*}{$\begin{array}{l}\text { Juveniles } \\
(n=64)\end{array}$} & $\begin{array}{l}\text { Liga nacio- } \\
\text { nal }\end{array}$ & 21 & 13,9 & $\begin{array}{l}16,6 \\
(0,8)\end{array}$ & $\begin{array}{l}15,9 \\
(0,3)\end{array}$ \\
\hline & $\begin{array}{l}\text { Juvenil } 1 \\
\text { División de } \\
\text { honor }\end{array}$ & 21 & 13,9 & & $\begin{array}{l}17,5 \\
(0,5)\end{array}$ \\
\hline & $\begin{array}{l}\text { Juvenil } 2 \\
\text { División de } \\
\text { honor }\end{array}$ & 22 & 14,6 & & $\begin{array}{l}16,9 \\
(0,4)\end{array}$ \\
\hline \multirow{3}{*}{$\begin{array}{l}\text { Categoría } \\
\text { absoluta } \\
(n=44)\end{array}$} & 3르 División & 23 & 15,2 & $\begin{array}{l}20,3 \\
(1,4)\end{array}$ & $\begin{array}{l}19,7 \\
(1,2)\end{array}$ \\
\hline & $\begin{array}{l}2^{a} \text { División } \\
\text { B }\end{array}$ & 21 & 13,9 & & $\begin{array}{l}21,0 \\
(1,1)\end{array}$ \\
\hline & Total & 151 & 100,0 & $17(2,3)$ & $17(2,3)$ \\
\hline
\end{tabular}

Fuente: elaboración propia.

\section{Variables e instrumentos}

\section{Edad, categoría deportiva y equipo}

Estas características fueron tomadas directamente de la ficha personal de cada jugador.

\section{Rendimiento deportivo medio}

Fue evaluado mediante la media sobre 16 observaciones de la actuación de cada deportista en 
los partidos de liga por parte del entrenador; la puntuación de rendimiento en cada actuación fue computada en función de una escala de 1 a 10 donde 1 significa el menor rendimiento posible y 10 el máximo. Esta misma metodología de valoración del rendimiento ha sido descrita por diferentes investigadores (García-Mas et al., 2003; Lane \& Chappell, 2001; Lowther, Lane \& Lane, 2002; Thelwell \& Maynard, 2003) en deportes como el fútbol, baloncesto y críquet, entre otros. La valoración subjetiva del rendimiento se basa en que los entrenadores, tras años de experiencia y de observación de jugadores, son capaces de tener una información comportamental relevante de los deportistas (Escudero, 1999; Escudero, Balagué \& García-Mas, 2002; Piedmont, Hill \& Blanco, 1999). Las valoraciones de los entrenadores representan una estimación apropiada de las capacidades de los jugadores, ya que están familiarizados con los criterios de rendimiento (Apitzsch, 1994; Vanyperen, 1994).

Motivación para el éxito, motivación para evitar el fracaso, competitividad rasgo

Estas variables fueron evaluadas con el cuestionario Competitividad-10. Se trata de un cuestionario breve de autoinforme con 10 preguntas sobre las motivaciones asociadas a la competitividad deportiva. El formato de respuesta es tipo Likert (1 = casi nunca, 2 = algunas veces, $3=$ a menudo). Se obtiene una puntuación independiente para la subescala Motivación de éxito (Me) y la subescala Motivación para evitar el fracaso (Mef) mediante la suma de los ítems y el cálculo de la media ponderada correspondiente a cada subescala. El indicador global del grado de competitividad rasgo se calcula mediante la diferencia entre Me y Mef (C $=\mathrm{Me}-\mathrm{Mef})$. Cuando la diferencia entre las dos dimensiones es positiva, por lo tanto superior para la $\mathrm{Me}$, la persona presenta una tendencia a tener alta motivación para competir. Las propiedades psicométricas (fiabilidad, validez: de constructo, concurrente, discriminante y predictiva) del cuestionario, están descritas en Remor (2007).

\section{Procedimiento}

La evaluación de los deportistas se realizó de forma grupal, antes del horario de inicio de los entrenamientos, en una sala de las instalaciones deportivas del club. Antes de realizar la evaluación, se les explicó a los jugadores que iban a participar en una investigación sobre comportamiento y deporte, que los resultados podían ser comentados individualmente, si lo deseaban, al terminar la temporada. La participación fue voluntaria, y dada la minoría de edad de algunos participantes en la investigación, para la cumplimentación de los cuestionarios se ha solicitado la autorización de los padres o del responsable. Los jugadores fueron citados una hora antes del inicio del entrenamiento. La información sobre el rendimiento deportivo (puntuación en cada partido) se ha recogido de la ficha de cada jugador, proporcionada por el entrenador. No se han "disponibilizado" los datos respecto al rendimiento del equipo de 2 2 a División 'B' $(n=21)$, por lo tanto no han sido incluidos en los respectivos análisis estadísticos. Para la realización de los análisis de datos se ha utilizado el programa estadístico SPSS versión 14.

\section{Resultados}

\section{Rendimiento deportivo de los futbolistas}

El rendimiento medio evaluado por la escala de 1 a 10 sobre dieciséis observaciones para todo el grupo fue de 7,3 (DT = 1,1; rango: 4 - 10). Para conocer la dispersión relativa en la variable rendimiento medio, se ha calculado el coeficiente de variación (dt/media) de dicha variable para cada participante, el resultado obtenido ha informado de un coeficiente de variación medio de 0,12 (varía entre 0 y 1 ) para toda la muestra. Por lo tanto, hay poca variación en las valoraciones individuales sobre el rendimiento hechas por los entrenadores, es decir, hay cierta estabilidad del rendimiento entre las observaciones y, consecuentemente, la media sobre las 16 observaciones es un buen indicador del rendimiento de cada individuo evaluado. 
Para valorar las posibles diferencias entre las categorías de equipo en relación con el rendimiento deportivo, se ha realizado un análisis de varianza (ANOVA) donde la categoría constituye la variable independiente y el rendimiento medio, la variable dependiente. Los resultados del ANOVA permiten valorar que ha habido diferencias significativas en el rendimiento medio en función de la categoría de equipo $(F(2,127)=55,069 ; p$ $=0.000$ ).

Las diferencias están presentes en la comparación de los tres grupos, observándose un aumento significativo en el rendimiento cuanto mayor la categoría deportiva (véase Tabla 2).

Considerando que edad y categoría deportiva están vinculadas, obsérvase una relación directa y significativa de la edad con el rendimiento medio (correlación de Pearson; $r=0,64 ; p=0.000$ ), es decir los individuos de mayor edad presentan puntuaciones más altas en el rendimiento.

Por otra parte, obsérvase una relación inversa entre el coeficiente de variación del rendimiento y la edad (correlación de Pearson; $r=-0,17 ; p=$ 0.042). La menor variación se presenta en el grupo de edad mayor de 19 años, y la mayor en el grupo entre 16 y 18 años $(F(2,127)=12,746 ; p=$ 0.000). Es decir, los futbolistas entre 16 y 18 años han presentado una mayor variación en el rendimiento observado y, por lo tanto, un rendimiento deportivo variable e inestable.

\section{La motivación de logro y la competitividad rasgo en relación con el rendimiento deportivo}

Los estadísticos descriptivos para las puntuaciones en el cuestionario Competitividad-10, en la presente muestra de futbolistas de competición fueron: $M=2.82(D T=0.18$; rango $2-3)$ para la motivación de éxito, $M=1.82(D T=0.40$; rango 1 - 3) para la motivación para evitar el fracaso y $M$ $=1(D T=0.48$; rango $-0.33-2)$ para el índice de competitividad.

No se ha observado una relación significativa entre las puntuaciones del cuestionario Competitividad-10 y la edad de los jugadores.

En la Tabla 3, se presentan los descriptivos de las puntuaciones del cuestionario Competitividad-10 en función del equipo a que pertenecen los jugadores, y se analizan el efecto del equipo sobre las puntuaciones en las dimensiones del cuestionario. Las diferencias se encuentran entre el equipo de Liga Nacional y Juvenil 1 División de Honor; al subir de categoría obsérvase menor miedo al fracaso y mayor competitividad rasgo.

Por otra parte, para valorar la relación entre motivación de logro (éxito/fracaso), competitividad rasgo y rendimiento se ha realizado un análisis de correlación de Pearson. Como se puede observar en la Tabla 4, al tener en cuenta comparaciones

TABLA 2

ANOVA: Categoría deportiva X Rendimiento Medio. Comparaciones múltiples post hoc: Prueba HSD de Tukey

\begin{tabular}{|c|c|c|c|}
\hline \multicolumn{2}{|r|}{ Categoría deportiva } & \multirow{2}{*}{$\begin{array}{c}\text { Diferencia de } \\
\text { medias (I-J) }\end{array}$} & \multirow{2}{*}{$p$} \\
\hline (I) & $(\mathrm{J})$ & & \\
\hline Cadete $(n=43 ; \mathrm{RM}=6,36)$ & Juveniles $(n=64 ; \mathrm{RM}=7,48)$ & $-1,11(*)$ & 0.000 \\
\hline Cadete & Categoría absoluta $(n=23 ; \mathrm{RM}=8,56)$ & $-2,22(*)$ & 0.000 \\
\hline Juveniles & Categoría absoluta & $-1,11(*)$ & 0.000 \\
\hline
\end{tabular}

Nota. Para 21 deportistas de la Categoría absoluta (Equipo 2a División 'B') todavía no están disponibles los datos sobre el rendimiento, por lo tanto no han sido incluidos en el análisis.

$\mathrm{RM}=$ Rendimiento medio del grupo de edad .

*La diferencia de medias es significativa al nivel 0.05.

Fuente: elaboración propia. 
TABLA 3

ANOVA. Dimensiones del cuestionario Competitividad-10 en función del equipo del jugador. Comparaciones múltiples post hoc. Prueba HSD de Tukey

\begin{tabular}{|c|c|c|c|c|c|}
\hline & Equipo & $n$ & Media & DT & ANOVA \\
\hline \multirow{8}{*}{$\begin{array}{l}\text { Motivación de } \\
\text { éxito }\end{array}$} & Cadete 1 Autonómica & 22 & 2,85 & 0,13 & \multirow{8}{*}{$\begin{array}{l}F(6,144)=1.525 ; p \\
=0.174\end{array}$} \\
\hline & Cadete 2 Autonómica & 21 & 2,73 & 0,26 & \\
\hline & Liga nacional & 21 & 2,80 & 0,18 & \\
\hline & Juvenil 1 División de honor & 21 & 2,87 & 0,14 & \\
\hline & Juvenil 2 División de honor & 22 & 2,82 & 0,19 & \\
\hline & 3a División & 23 & 2,81 & 0,17 & \\
\hline & 2-a División B & 21 & 2,86 & 0,14 & \\
\hline & Total & 151 & 2,82 & 0,18 & \\
\hline \multirow{8}{*}{$\begin{array}{l}\text { Motivación para } \\
\text { evitar el fracaso }\end{array}$} & Cadete 1 Autonómica & 22 & 1,87 & 0,39 & \multirow{8}{*}{$\begin{array}{l}F(6,144)=2.323 ; p \\
=0,036^{*} \\
\text { Las diferencias se en- } \\
\text { cuentran entre Liga } \\
\text { nacional y Juvenil } 1 \\
(\text { Post hoc, HSD de } \\
\left.\text { Tukey, } p=0,047^{*}\right)\end{array}$} \\
\hline & Cadete 2 Autonómica & 21 & 1,77 & 0,41 & \\
\hline & Liga nacional (-) & 21 & 2,04 & 0,40 & \\
\hline & Juvenil 1 División de honor (-) & 21 & 1,67 & 0,40 & \\
\hline & Juvenil 2 División de honor & 22 & 1,90 & 0,49 & \\
\hline & $3^{\text {a }}$ División & 23 & 1,77 & 0,30 & \\
\hline & 2a División B & 21 & 1,69 & 0,33 & \\
\hline & Total & 151 & 1,82 & 0,40 & \\
\hline \multirow{8}{*}{$\begin{array}{l}\text { Competitividad } \\
\text { rasgo }\end{array}$} & Cadete 1 Autonómica & 22 & 0,98 & 0,46 & \multirow{8}{*}{$\begin{array}{l}F(6,144)=2.191 ; p \\
=0.047 * \\
\text { Las diferencias se en- } \\
\text { cuentran entre Liga } \\
\text { nacional y Juvenil } 1 \\
\text { (post hoc, HSD de } \\
\text { Tukey, } p=0.047^{*} \text { ) }\end{array}$} \\
\hline & Cadete 2 Autonómica & 21 & 0,95 & 0,51 & \\
\hline & Liga nacional (-) & 21 & 0,75 & 0,50 & \\
\hline & Juvenil 1 División de honor (-) & 21 & 1,19 & 0,42 & \\
\hline & Juvenil 2 División de honor & 22 & 0,91 & 0,58 & \\
\hline & $3^{\text {a }}$ División & 23 & 1,04 & 0,41 & \\
\hline & 2aㅡ División B & 21 & 1,17 & 0,38 & \\
\hline & Total & 151 & 1,00 & 0,48 & \\
\hline
\end{tabular}

$*_{p}<0,05 ;(-)$ equipos entre los cuales hay diferencias significativas.

Fuente: elaboración propia.

entre individuos en el mismo nivel de categoría deportiva y edades similares, las relaciones entre motivación de logro, competitividad rasgo y rendimiento deportivo, aumentan; excepto para la categoría de juveniles donde el nivel de motivación y competitividad no está relacionado con el rendimiento que presentan. 
TABLA 4

Correlaciones de Pearson entre motivación de logro (Me, Mef), competitividad y rendimiento medio

\begin{tabular}{lccc}
\hline \multicolumn{1}{c}{ Rendimiento Medio } & Motivación de éxito & Motivación para evitar el fracaso & Competitividad rasgo \\
\hline Todas las categorías $(\mathrm{n}=130)$ & $0,219 *(0.012)$ & $-0,091(\mathrm{n} . \mathrm{s})$. & $0,160^{+}(0.070)$ \\
Cadetes $(\mathrm{n}=43)$ & $0,373 *(0.014)$ & $-0,258^{+}(0.094)$ & $0,385 *(0.011)$ \\
Juveniles $(\mathrm{n}=64)$ & 0,057 (n.s.) & $0,068(\mathrm{n} . \mathrm{s})$. & $-0,039$ (n.s.) \\
Categoría absoluta $(\mathrm{n}=23)$ & $0,495 *(0.016)$ & $-0,432(0.040)$ & $0,525 *(0.010)$ \\
\hline
\end{tabular}

Nota. Para 21 deportistas de la Categoría absoluta (Equipo 2ª División 'B’) todavía no están disponibles los datos sobre el rendimiento, por lo tanto no han sido incluidos en el análisis.

$* p<0.05 ;{ }^{+} p<0.10$ (n.s.); (n.s.) $=$ no significativo

Fuente: elaboración propia.

\section{Discusión}

El presente estudio identificó una relación entre rendimiento deportivo y el nivel de la categoría deportiva de deportistas de competición (fútbol), donde a mayor nivel de profesionalización se observa mayor rendimiento medio. Aunque este resultado parezca obvio, podemos entender esta relación como la expresión de la dimensión reactiva y proactiva de la personalidad (MorenoJiménez, 2007). Es decir, el contexto o situación ejerce un efecto sobre la conducta, de forma que si el contexto es de logro tiende a observarse mayor expresión de la conducta de rendimiento optimo y de competitividad (dimensión reactiva); por otra parte - como planteaban Franken et al. (1994) y Weinberg y Gould (1996) -, las personas altamente competitivas tiendan a buscar situaciones de competición y están motivadas para obtener éxito en ellas, lo que es un reflejo de la dimensión proactiva de la personalidad, la capacidad para organizar la conducta atendiendo a objetivos y metas que el individuo se plantea obtener.

Otro aspecto interesante de este trabajo fue contar como medida de rendimiento: la media entre dieciséis observaciones de la actuación del deportista. Tener en cuenta varias observaciones de la conducta deportiva en situaciones reales, parece ofrecer una información más precisa del rendimiento medio real del deportista, tal y como se ha reflejado a través del coeficiente de variación calculado sobre la medida de rendimiento. Se ha identificado un coeficiente de variación bajo entre observaciones, que permite valorar que el rendimiento medio fue representativo de las diferentes observaciones.

Además, al calcular el coeficiente de variación para valorar si el rendimiento medio puntuado, fue representativo del rendimiento general del deportista, se está accediendo a una información sobre la estabilidad del rendimiento del deportista.

Por otra parte, hemos observado un efecto de la categoría deportiva sobre el rendimiento evaluado, a medida que se sube de categoría el rendimiento deportivo es mayor. Estos resultados confirman hallazgos de investigaciones anteriores (p. ej., Gee et al., 2007). Así mismo, y en la línea de lo anterior, se ha identificado una relación lineal directa entre edad del deportista y rendimiento medio, a medida que aumenta la edad observase mayor rendimiento. No obstante, un aspecto que cabe matizar en esta relación es que hay un efecto de la edad sobre la calidad del rendimiento. Los análisis presentados permiten identificar que el grupo de edad intermedia -entre 16 y 18 años-ha presentado menor estabilidad en las puntuaciones de rendimiento, por lo tanto mayor variación media del rendimiento. En otras palabras, en algunas observaciones han presentado alto rendimiento y en otras bajo rendimiento. Se pueden proponer diferentes hipótesis explicativas para dichos resultados, por una parte, podría estar relacionado con 
la etapa evolutiva de este grupo de edad, donde quizás la mayor diversidad de intereses personales (o factores que desconocemos) podría estar afectando la capacidad de concentración y motivación de estos deportistas; o aún, por otra parte, puede que los individuos del grupo de edad en categoría intermedia perciban el futuro como incierto en términos de profesionalización en el fútbol, la alta presión para obtener resultados y poder pasar a categorías superiores podrían estar afectando igualmente la capacidad de atención, concentración y motivación de dichos deportistas. Futuros trabajos podrían intentar abordar esta cuestión haciendo un seguimiento longitudinal de los deportistas y valorando cualitativamente sus percepciones sobre estos resultados, con el objetivo de poder elucidar esta cuestión.

En relación a las dimensiones de la motivación de logro (Me, Mef) y la competitividad rasgo, se observa que las puntuaciones medias del presente estudio en varones deportistas de competición (fútbol) han sido superiores a las descritas por Remor (2007) en varones deportista universitarios (no profesionales). Lo que parece corroborar la hipótesis de que a mayor nivel de profesionalización en el deporte, mayor competitividad disposicional. Estos resultados van en la línea de otros trabajos (p. ej., Gill, 1986; Gill, Dzewaltowski \& Deeter, 1988; Hellandsig, 1998; Martin \& Ecklund, 1994).

Y como han puesto de manifiesto estudios anteriores (p. ej., Beaudoin, 2006; Remor, 2007) no se ha observado una relación entre competitividad rasgo y la edad de los deportistas. Es muy probable que otras variables externas o situacionales sean más determinantes de la disposición a competir que la edad del individuo.

Por otra parte, se han observado diferencias en la motivación para evitar el fracaso y la competitividad rasgo en función de la categoría deportiva; no obstante, estas diferencias han estado presentes solamente entre las categorías Liga Nacional y Juvenil (División de Honor). La categoría de mayor nivel ha presentado menor miedo al fracaso y mayor competitividad. Se entiende que la asignación a un equipo de mayor nivel puede contribuir a una percepción de mayor competencia y autoeficacia personal por parte del jugador, generando un beneficio psicológico para el deportista.

Los resultados del estudio han permitido identificar también que la motivación de éxito y la competitividad rasgo están asociadas a mayor rendimiento deportivo, mientras que la motivación para evitar el fracaso se ha asociado negativamente con el rendimiento. Estos resultados van en la línea de otras investigaciones previas (p. ej., Gee et al., 2007; Gould et al., 2002; Martin \& Ecklund, 1994; Remor, 2007; Sáenz-López et al., 2007).

Por último, también la categoría deportiva parece tener un efecto mediador sobre estas relaciones. Al valorar las correlaciones entre las variables - Me, Mef, competitividad rasgo y rendimiento medio - en función de la categoría deportiva, se puede identificar que la categoría de cadetes y la categoría absoluta mantienen la asociación entre dichas variables, mientras que para la categoría de juveniles no se observa ninguna asociación significativa; en otras palabras, parece que para esta categoría hay otras variables que pueden explicar mejor el rendimiento que el tipo de motivación y el nivel de competitividad. Futuros estudios podrían ocuparse de comprender mejor estos resultados, considerando si ello se debe a un efecto de las situaciones o de aspectos intrapersonales, o aun relacionado con aspectos del desarrollo evolutivo.

\section{Referencias}

Apitzsch, E. (1994). La personalidad del jugador de fútbol de élite. Revista de Psicología del Deporte, 6, 89-98.

Beaudoin, C. M. (2006). Competitive orientations and sport motivation of professional women football players: An internet survey. Journal of Sport Behavior, 29, 201-212.

Carlstedt, R. (2004). Critical moments during competition: A mind-body model of sport performance when it counts the most. London: Taylor \& Francis.

Cashdan, E. (1998). Are men more competitive than women? British Journal of Social Psychology, 37, 213-229. 
Dosil, J. (2003). Tendencias de la investigación en Psicología de la Actividad física y del deporte. EduPsykhé, 2, 147-164.

Escudero, J. (1999). Uso y conocimiento de variables psicológicas y evaluación de comportamientos deportivos de jugadores por parte de los entrenadores de baloncesto orientados al rendimiento. Tesis doctoral no publicada, Departamento de Psicología, Universitat Illes Balears, España.

Escudero, J., Balagué, G. \& García-Mas, A. (2002). Comportamientos que influyen en el rendimiento deportivo de jugadores de baloncesto desde el punto de vista de los entrenadores. Psicothema, 14, 34-38.

Franken, R., Hill, R. \& Kierstead, J. (1994). Sport interest as predicted by the personality measures of competitiveness, mastery, instrumentality, expressivity, and sensation seeking. Personality and Individual Differences, 17, 467-476.

García-Mas, A., Aguado, F. J., Cuartero, J., Calabria, E., Jiménez, R. \& Pérez, P. (2003). Sueño, descanso y rendimiento en jóvenes deportistas de competición. Revista de Psicología del Deporte, 12 (2), 181-195.

García-Naveira, A. \& Remor, E. (2008, marzo). Diferencias en competitividad en función del nivel competitivo en jugadores de fútbol adultos. XI Congreso Nacional de Psicología de la Actividad Física y el Deporte, XI Congreso Andaluz y III Iberoamericano. Federación Española de Psicología del Deporte, Asociación de Psicología del Deporte de Andalucía y Universidad Pablo de Olavide, Sevilla, España.

Gee, C., Dougan, R., Marshall, J. \& Dunn, L. (2007, June). Using a normative personality profile to predict success in the National Hockey League (NHL): A 15-year longitudinal study. North American Society for the Psychology of Sport and Physical Activity Conference, San Diego, CA, U.S.A.

Gill, D. L. (1986). Competitiveness among females and males in physical activity classes. Sex Roles, 15, 233-247.

Gill, D., Dzewaltowski, D. \& Deeter, T. (1988). The relationship of competitiveness and achievement orientation to participation in sport and nonsport activities. Journal of Sport and Exercise Psychology, 10, 139-150.
Gill, D. L., Williams, L., Dowd, D. A., Beaudoin, C. M. \& Martin, J. J. (1996). Competitive orientations and motives of adult sport and exercise participants. Journal of Sport Behavior, 19, 307-318.

Gould, D., Dieffenbach, K. \& Moffett, A. (2002). Psychological characteristics and their development in olimpic champions. Journal of Applied Sport Psychology, 14, 172-204.

Hellandsig, E. T. (1998). Motivational predictors of high performance and discontinuation in different types of sports among talented teenage athletes. International Journal of Sport Psychology, 29, 27-44.

Houston, J., Harris, P., McIntire, S. \& Francis, D. (2002). Revising the competitiveness index using factor analysis. Psychological Reports, 90, 31-34.

Houston, J., Harris, P., Moore, R., Brummett, R. \& Kmetani, H. (2005). Competitiveness among Japanese, Chinese, and American undergraduate students. Psychological Reports, 97, 205-212.

Jarvis, M. (2005). Sport Psychology. A Student Handbook (ed. rev.). London: Taylor \& Francis.

Lane, A. M. \& Chappell, R. H. (2001). Mood and performance relationships at the World Student Games Basketball Competition. Journal of Sport Behavior, 24, 182-196.

Lowther, J., Lane, A. \& Lane, H. (2002). Self-efficacy and psychological skills during The Amputee Soccer World Cup. Athletic Insight, 4, 23-34. Recuperado el 02 de diciembre de 2008, de http:// www.athleticinsight.com/Vol4Iss2/SoccerSelfEfficacy.htm

Martens, R. (1975). Social Psychology of Sport. New York: Harper \& Row.

Martin, J. \& Ecklund, R. (1994). The relationships among competitiveness, age and ability in distance runners. Journal of Sport Behavior, 17, 258-266.

Moran, A. P. (2004). Sport and exercise Psychology: A critical introduction. New York: Routledge.

Moreno-Jiménez, B. (2007). Psicología de la Personalidad. Procesos. Madrid: Thomson.

Núñez, J. L. (1998). Personalidad y motivación del jugador de tenis. Tesis doctoral no publicada, Centro Superior de Formación del Profesorado, Universidad de las Palmas de Gran Canaria, España.

Piedmont, R., Hill, D. \& Blanco, S. (1999). Predicting athletic performance using the five-factor model 
of personality. Personality and Individual Differences, 27, 769-777.

Remor, E. (2007). Propuesta de un cuestionario breve para la evaluación de la competitividad en el ámbito deportivo: Competitividad-10. Revista de Psicología del Deporte, 16, 167-183.

Ruiz, R. (2006). Predicción del resultado deportivo en judokas cadetes de competición aplicando las dimensiones del cuestionario de personalidad BFQ. Revista Iberoamericana de Psicología del Ejercicio y del Deporte, 1, 69-88.

Sáenz-López, P., Jiménez, A., Jiménez, F. \& Ibáñez, S. (2007). La autopercepción de las jugadoras de baloncesto expertas respecto a sus procesos de formación. Cultura, Ciencia y Deporte, 3, 35-41.

Singer, R., Hausemblas, H. \& Jannelle, C. (2001). Prologue: A brief history of research in Sport Psychology. In R. Singer, H. Hausemblas \& C. Janelle (Eds.), Handbook of Sport Psychology (pp. 13-19). New York: John Wiley \& Sons.
Thewell, R. C. \& Maynard, I. W. (2003). The effects of mental skills package on "repeatable good performance" in cricketers. Psychology of Sport and Exercise, 4, 377-396.

Vanyperen, N. W. (1994). Estrés psicosocial, apoyo parental, y rendimiento en jóvenes futbolistas con talento. Revista de Psicología del Deporte, 6, 119-138.

Vealey, R. S. (1992). Personality and sport: A comprehensive view. En T. S. Horn (Ed.), Advances in Sport Psychology (pp. 25-59). Champaign, IL: Human Kinetics.

Vealey, R. S. (1994). Current status and prominent issues in Sport Psychology interventions. Medicine and Science in Sports and Exercise, 26, 495-502.

Viadé, A. (2003). Psicología del rendimiento deportivo. Barcelona: Editorial UOC.

Weinberg, R. \& Gould, D. (1996). Fundamentos de Psicología del deporte y el ejercicio. Barcelona: Ariel. 
